\title{
DEFORMATIONS AND SPLITTINGS OF A BUBBLE IN A TWO-DIMENSIONAL FLUIDIZED BED EXPERIMENTAL RESULTS*
}

\author{
RYOZo TOEI, RYUICHI MATSUNO**, MORIHIRO OICHI \\ AND KATSUNOBU YAMAMOTO*** \\ Department of Chemical Engineering, Kyoto University, Kyoto
}

\begin{abstract}
The behavior of deformations and splittings of a single bubble was studied experimentally in a two-dimensional fluidized bed. It was found that small downward cusps were generated at the bubble surface near the stagnation point and that they grew and necessarily caused bubble deformations and, frequently, bubble splittings. The authors call these cusps "disturbances".

The frequencies of disturbances and splittings were observed and the following results were found. 1) About $4 \sim 8$ disturbances were generated per second almost near the stagnation point, and they ran along the edge of a bubble to be lost near the wake; 2) about 14 30 percent of the disturbances also caused bubble splittings; 3) there were two types of splittings; 4) the frequencies of distrubances and splittings decreased with increase of particle diameter; and 5) neither frequency depended much on bubble diameter.
\end{abstract}

\section{Introduction}

Deformations and splittings of a bubble occur repeatedly during its rising in a gas-solid fluidized bed. From experiments with gas exchange between a bubble and the continuous phase using brown $\mathrm{NO}_{2}$ gas $^{7)}$, it was confirmed that when a bubble coalesces with another or splits, a large amount of $\mathrm{NO}_{2}$ gas flows out of the bubble cloud. Therefore it seems that bubble coalescences and splittings play an important role in transport phenomena.

The mechanism of bubble coalescence and the gas transfer mechanism in this case have been studied frequently ${ }^{2,3,6,7)}$, but there have been very few studies concerning bubble splitting ${ }^{4}$. In this paper, the behavior of bubble deformations and splittings was studied experimentally in a two-dimensional bed and partly in a three-dimensional one.

\section{Experimental Equipment and Procedures}

A two-dimensional fluidized bed $(25 \mathrm{~cm}$ wide, $2.3 \mathrm{~cm}$ deep and $150 \mathrm{~cm}$ high) made of polymethylmethacrylate was used. The insides of the walls were covered with glass plates to keep off the electrostatic effect of glass particles. The distributor was a perforated plate, and the calming section was made

* Received on February 18, 1974

Presented at the 7th Autumn Meeting of The Soc. of Chem. Engrs., Japan, October 1973

** Dept. of Food Sci. and Tech., Fac. of Agri., Kyoto Univ. *** Fuji Photo Film Co., Ltd.

干606 京都市左京区吉田本町

京都大学工学部化学工学科 尾市守弘 of a packed bed $\left(7 \mathrm{~cm}\right.$ high) of about $0.2 \mathrm{~mm}^{\phi}$ glass beads. The bed was maintained close to the minimum fluidization state by air. A single bubble was injected through a nozzle by a solenoid valve connected to the pressure vessel. The nozzle was installed $11 \mathrm{~cm}$ above the distributor. The diameter of the injected bubble was 3 to $13 \mathrm{~cm}$ and was controlled by the pressure in the vessel.

The bubble was photographed by a 16-mm cine camera at speed of 64 frames per second with counterlight. By rotating the camera in synchronization with the bubble rising velocity, the buble was kept always near the center of the pictures. Three kinds of glass beads, listed in Table $\mathbf{1}$, were used as fluidized particles.

The authors ${ }^{5)}$ formerly photographed a single bubble or continuous bubbles in a three-dimensional bed $(10 \mathrm{~cm} \times 10 \mathrm{~cm})$ using $\mathrm{X}$-ray movies. These films were used as reference in the case of three-dimensional bubbles.

\section{Results and Discussion}

\begin{tabular}{|c|c|c|c|}
\hline particle [glass beads] & A & B & $\mathrm{C}$ \\
\hline Tyler mesh & $-35+48$ & $-42+65$ & $-60+80$ \\
\hline$\overline{d_{p}} \quad[\mathrm{~mm}]$ & 0.356 & 0.280 & 0.210 \\
\hline $\begin{array}{l}u_{m f} \\
\text { fraction of splittings of }\end{array}$ & 9.86 & 6.15 & 3.00 \\
\hline type 2) in all splittings & 0.141 & 0.199 & 0.294 \\
\hline$[1 / \mathrm{sec}]$ & 3.85 & 5.39 & 7.73 \\
\hline$[1 / \mathrm{sec}]$ & 0.524 & 1.03 & 2.34 \\
\hline$\left(\overline{f_{s, t}} / \overline{f_{d, t}}\right)$ & 0.136 & 0.191 & 0.302 \\
\hline
\end{tabular}




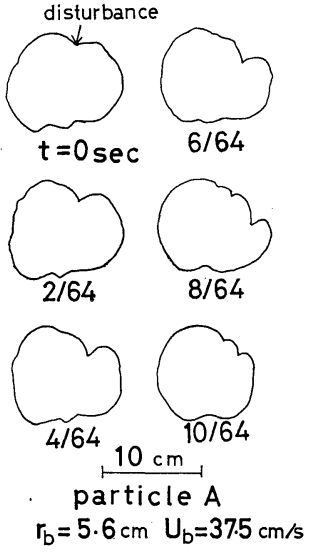

Fig. 1 Bubble deformation

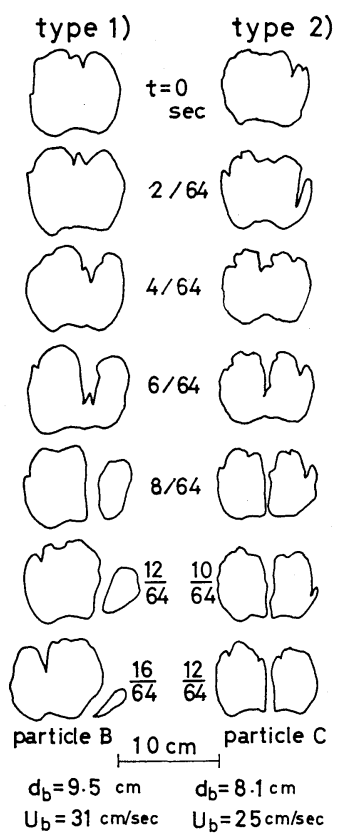

Fig. 2 Both types of bubble splittings

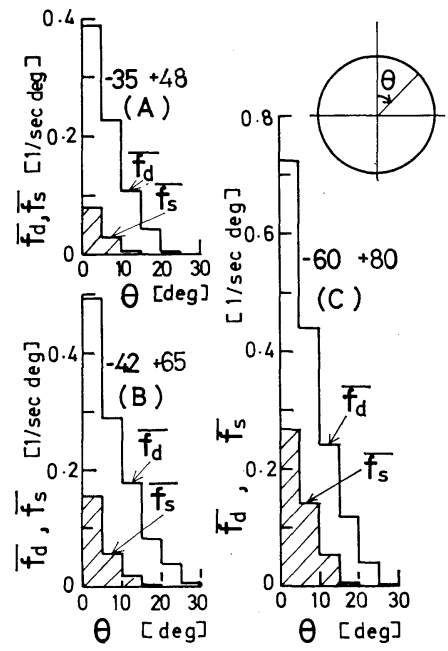

Fig. 3 Distributions of average frequencies of disturbances $\overline{f_{d}}$ and splittings $\overline{f_{s}}$

\section{Behavior of bubble deformations and splittings}

The behavior of a bubble deformation was traced by analyzing photographic films. As Rowe et al. pointed out ${ }^{4}$, small downward cusps are generated at the upper surface of a bubble, the surface becomes uneven, and these cusps grow rapidly into long fingers. In the present paper, the authors call these cusps "disturbances". The disturbances tend to originate near the stagnation point of a bubble, running along the edge to be lost near the wake, and consequently they cause a bubble deformation. An example is shown in Fig. 1. About 0.2 0.3 second is necessary for a disturbance generated near the stagnation point to be lost near the wake. It is found that an initially nearly cylindrical bubble becomes in general vertically elongated after deformation.

In some cases, a disturbance grows so fast relative to its lateral movement that it divides the bubble in two. There are two types of bubble splittings: 1) a small part of a bubble is sliced off but almost immediately re-absorbed, and 2) a bubble is divided in two nearly equal-size bubbles which rise independently of each other. In Fig. 2, both types of bubble splittings are shown. The fraction of type 2 in all splittings is shown in Table 1. As particles become coarse, the fraction becomes small and therefore the occurrence of splittings of type 2 becomes difficult, together with the decrease of the frequency of splittings as mentioned later. In this experiment, the number of total splittings measured was 140 to 550 for each particle diameter.

Bubble splittings are not peculiar phenomena in two-dimensional bubbles, but as shown in the X-ray pictures by Rowe et al. ${ }^{4}$, they also happen in threedimensional bubbles. The authors confirmed that both types of bubble splittings were seen in a threedimensional bed by observing films ${ }^{5}$ which had been photographed by using $\mathrm{X}$-rays, though the pictures of a bubble or continuous bubbles were not clear enough to analyze the films quantitatively since the X-ray intensity was not very strong.

\section{Frequency of disturbances and splittings}

The disturbance necessarily causes deformation of a bubble and also frequently causes splitting. The frequency of disturbances was measured. The number of disturbances newly generated was counted in each measuring section of five degrees of the angle along the periphery of a bubble. The frequency of disturbances per degree at the angle $\theta$ from the stagnation point was calculated by dividing their number by the measuring time and the width of the measuring section (5 degrees). The range of bubble diameter was 3 to $13 \mathrm{~cm}$. The number of total disturbances counted for each particle diameter was 620 to 1050 . The frequency of splittings (i.e. that of 
disturbances which caused bubble splitting) was also measured in the same way as mentioned above. Since the distribution of the frequency of disturbances or splittings against the angle does not depend so much on the bubble diameter, as was checked later, average values of both frequencies for various bubble diameters are employed.

In Fig. 3, the average frequencies of disturbances $\overline{f_{d}}$ [number/sec $\cdot$ degree] and splittings $\overline{f_{s}}$ are shown. In the figure, the hatched parts show $\overline{f_{s}}$. The effect of particle diameter on $\overline{f_{d}}$ and $\overline{f_{s}}$ is remarkable. As particle diameter becomes smaller, $\overline{f_{d}}$ and $\overline{f_{s}}$ become larger. However, the relations to the angle $\theta$ are similar in all cases. The value of $\overline{f_{d}}$ is largest at the stagnation point and decreases rapidly with increase of the angle $\theta$, and as for splittings, the tendency is nearly the same as for disturbances. It is also found that about 75 percent of disturbances are generated at the section of 20 degrees holding the stagnation point as the center, and especially for disturbances which divide a bubble, about 90 percent of them are generated at the section. The reason for this phenomenon is considered to be as follows. Let's consider the forces acting on particles moving along the bubble surface. The centrifugal force acting on particles is proportional to the square of the tangential velocity of particles and therefore proportional to $\sin ^{2} \theta^{11}$, while the centripetal force is the cosine component of gravitational force. Therefore the ratio of centrifugal to centripetal force, which is considered as the measure of the resisting force against the disturbance, increases as the angle $\theta$ increases, so that the frequency of the disturbances decreases with increase of $\theta$.

In Fig. 4, the ratio $\left(\overline{f_{d}} / \overline{f_{d, t}}\right)$ and $\left(\overline{f_{s}} / \overline{f_{d, t}}\right)$ are plotted against the angle $\theta$. It is said that for disturbances $\left(\widetilde{f_{d}} / \overline{f_{d, t}}\right)$ does not depend on particle diameter, while for splittings $\left(\overline{f_{s}} / \overline{f_{d, t}}\right)$ is affected somewhat by particle diameter because of the difference of $\left(\overline{f_{s, t}} / \overline{f_{d, t}}\right)$ mentioned later.

The values of $\overline{f_{d, t}}$ and $\overline{f_{s, t}}$ are shown in Table 1 and Fig. 5 against $u_{m f}$. It is also shown that particle diameter affects frequencies. The values of $\overline{f_{d, t}}$ and $\overline{f_{s, t}}$ decrease with increase of $u_{m f}$. The dependency of $\overline{f_{s, t}}$ on $u_{m f}$ is nearly two times larger than that of $\overline{f_{d, t}}$ within this experimental range. In Table 1, the ratio $\left(\overline{f_{s, t} t} / \overline{f_{d, t}}\right)$, that is, the fraction of disturbances causing bubble splitting in all disturbances, is also shown. As particle diameter increases, $\left(\overline{f_{s, t}} / \overline{f_{d, t}}\right)$ decreases and therefore, together with the decrease of $\overline{f_{d, t}}, \overline{f_{s, t}}$ decreases sharply. It is considered that this phenomenon comes from the difference of magnitude of gas circulation within the bubble cloud, which has the opposite force for the growing up of disturbances. Since the magnitude of gas circulation is

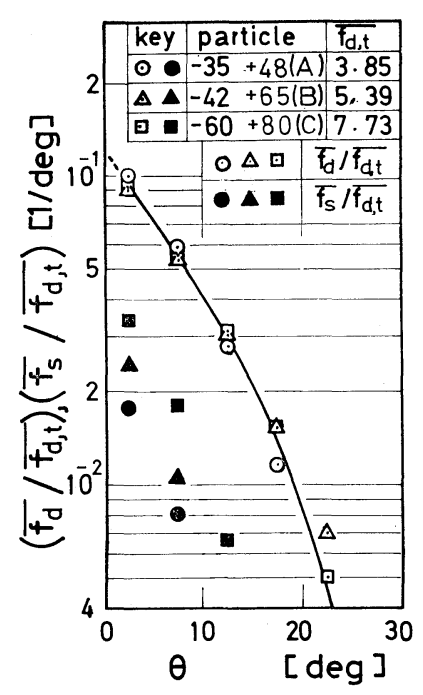

Fig. 4 Distributions of ratios $\left(\overline{f_{d}} / \overline{f_{d, t}}\right)$ and $\left(\overline{f_{s}} / \overline{f_{d, t}}\right)$ against angle $\theta$

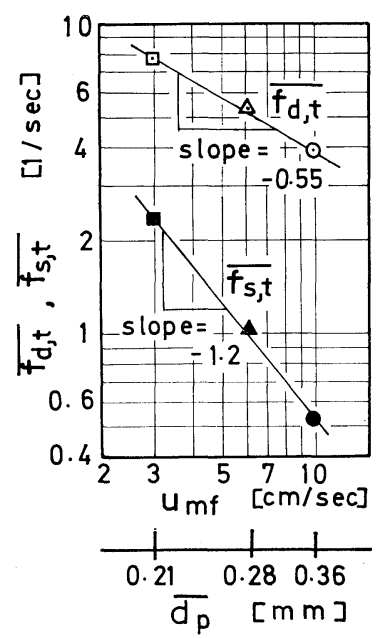

Fig. 5 Effect of the particle diameter on average total frequencies of disturbances $\overline{f_{d, t}}$ and splittings $\overline{f_{s, t}}$

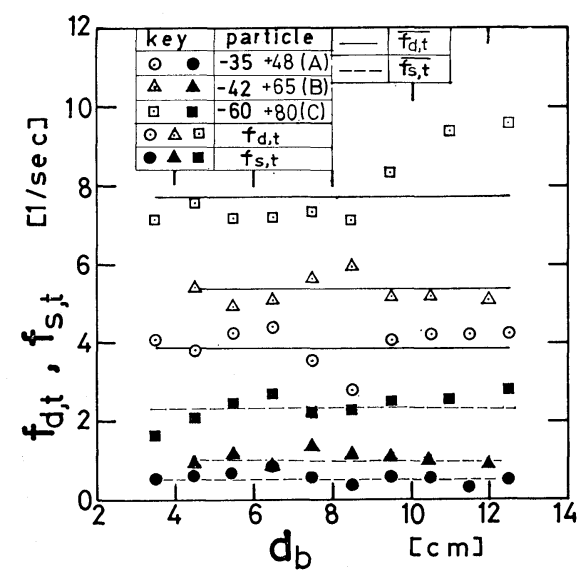

Fig. 6 Effect of the bubble diameter on total frequencies of disturbances $f_{d, t}$ and splittings $f_{s, t}$

proportional to $u_{m f}{ }^{11}$, a bubble in a bed of coarser particles has a stronger resisting force per unit surface against disturbances and suffers less from their 
effects.

As described before, since the bubble diameter does not much affect either frequency, the average values of these $\overline{f_{d}}, \overline{f_{s}}$ and $\overline{f_{d, t}}, \overline{f_{s, t}}$ for various bubble diameters could be taken. Finally, the effect of bubble diameter is checked here. In Fig. 6, the total frequencies of disturbances $f_{d, t}$ and splittings $f_{s, t}$ are shown against bubble diameter. For the particle $\mathrm{A}$ and $\mathrm{B}$, both frequencies $f_{d, t}$ and $f_{s, t}$ are almost independent of bubble diameter, but for the finest particle $C$, it seems that the value of $f_{d, t}$ increases gradually with bubble diameter when the diameter becomes larger than $9 \mathrm{~cm}$.

\section{Conclusion}

The behavior of deformations and splittings of a single bubble was studied in a two-dimensional fluidized bed as the first step. It was found that small downward cusps were generated, grew and necessarily caused bubble deformations and, frequently, bubble splittings. The authors call these cusps "disturbances".

The frequencies of disturbances and splittings were observed and the following results were found: 1) most disturbances were generated near the stagnation point at the frequency of about $4 \sim 8$ per second, and the frequency of disturbances or splitting at the angle $\theta$ decreased sharply with increase of the angle from stagnation point; 2) about $14 \sim 30$ percent of disturbances caused not only bubble deformations but also bubble splittings; 3 ) there were two types of splittings, that is, those of a part of the bubble and those into nearly two equal-size bubbles, and the percentage of the latter was $14 \sim 30$; 4) the frequency of disturbances or splittings decreased with increase of particle diameter; and 5) neither frequency depended much on bubble diameter.

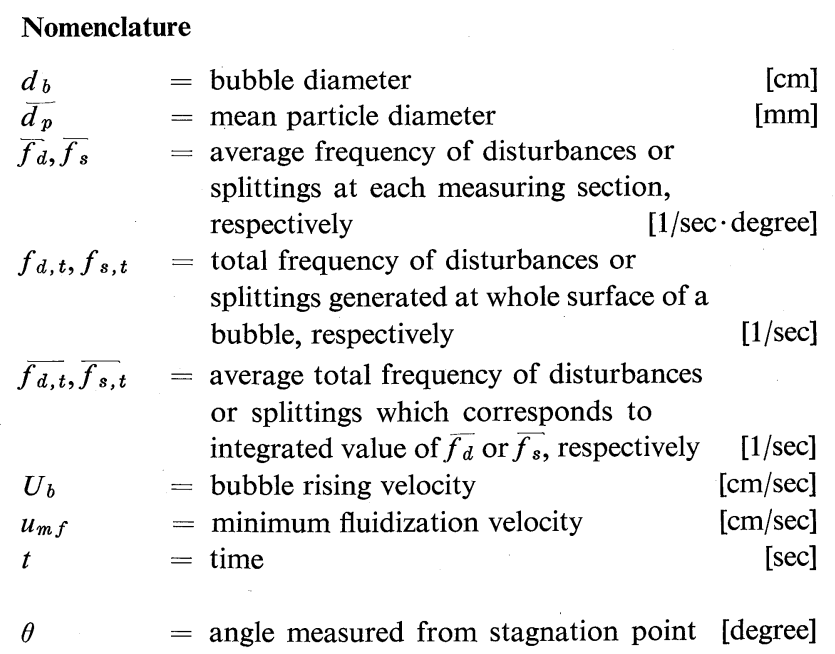

\section{Literature Cited}

1) Davidson, J. F. and D. Harrison: "Fluidized Particles", Cambridge University Press (1963)

2) Miwa, K., S. Mori, T. Kato and I. Muchi: Kagaku Kōgaku, 35, 770 (1971)

3) Mori, S. and I. Muchi: J. Chem. Eng. Japan, 5, 251 (1972)

4) Rowe, P. N. and B. A. Partridge: Trans. Instn. Chem. Engrs., 43, 157 (1965)

5) Toei, R., R. Matsuno, H. Kojima, Y. Nagai, K. Nakagawa and S. Yu: Kagaku Kögaku, 29, 851 (1965)

6) Toei, R., R. Matsuno, T. Sumitani and T. Mori: ibid., 31 861 (1967)

7) Toei, R., R. Matsuno, H. Nishitani and T. Imamoto: ibid., 33, 668 (1969) 